\title{
DESENVOLVIMENTO DA VERSÃO EM PORTUGUÊS DO NUTRISCORE
}

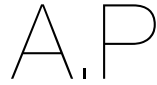

ARTIGOPROFSSIONAL

1 Faculdade de Ciências da Nutrição e Alimentação da Universidade do Porto, Rua do Campo Alegre,

n. ${ }^{8} 823$,

4150-180 Porto, Portugal

2 Unidade Local de Saúde do Alto Minho, Entidade Pública Empresarial (EPE) Hospital de Santa Luzia, Estrada de Santa Luzia, 4904-858 Viana do Castelo, Portugal

Endereço para correspondência Teresa Amaral Rua do Campo Alegre, n. ${ }^{\circ} 823$ 4150-180 Porto, Portugal. amaral@fcna.up.p

Histórico do artigo:

Recebido a 13 de maio de 2020 Aceite a 30 de junho de 2020

\section{DEVELOPMENT OF THE PORTUGUESE VERSION OF} NUTRISCORE

Joana FG Mendes'; Elisa Ruivo²; Ana Simas'; Teresa F Amaral'

\section{RESUMO}

O Nutriscore é a única ferramenta de rastreio da desnutrição desenhada especificamente para doentes oncológicos em ambulatório. Dado que não existe uma versão em português, o objetivo deste estudo foi produzir uma tradução Portuguesa validada. Foram seguidas as recomendações da International Society for Pharmacoeconomics and Outcomes Research, tendo-se cumprido os dez passos: preparação, tradução, reconciliação, retrotradução, revisão da retrotradução, harmonização, avaliação da compreensão, revisão, finalização e produção do relatório final. A ferramenta foi aplicada a uma amostra de 46 doentes com patologia oncológica, seguida em ambulatório. Conclui-se, assim, que a versão em Português do Nutriscore mantém os conceitos e equivalência da ferramenta original, é de fácil compreensão e aplicação, com boa aceitabilidade e validade aparente. Esta versão terá utilidade para determinar o risco de desnutrição em doentes oncológicos seguidos em ambulatório em Portugal e eventualmente noutros locais onde seja usada a língua Portuguesa.

\section{PALAVRAS-CHAVE}

Desnutrição, Nutriscore, Oncologia, Rastreio nutricional

\section{ABSTRACT}

Nutriscore is the only nutritional screening tool developed specifically for oncological outpatients. Due to the absence of a Portuguese version, the aim of this study was to create a Portuguese validated translation. The ten steps of the International Society for Pharmacoeconomics and Outcomes Research guideline were followed: preparation, forward translation, reconciliation, back translation, back translation review, harmonization, cognitive debriefing, review of the cognitive debriefing results and finalization, proofreading and final report. The screening tool was applied to 46 oncological outpatients by six health care providers. It can be concluded that the Portuguese version of Nutriscore maintains the concepts and equivalence of the original tool, is easy to understand and apply, with good acceptability and face validity. This version will be useful for determining the risk of undernutrition in outpatient oncological patients in Portugal and eventually in other settings where the Portuguese language is used.

KEYWORDS

Undernutrition, Nutriscore, Oncology, Nutritional screening

\section{INTRODUÇÃO}

O Nutriscore é a única ferramenta de rastreio da desnutrição desenhada especificamente para doentes oncológicos em ambulatório. Esta foi desenvolvida em 2016 por Lorena Arribas e col. (1), que estabeleceram como objetivos que esta possa ser aplicada por qualquer profissional de saúde, seja precisa, rápida e simples e, através da sua aplicação, ser possível identificar os casos de risco nutricional, minimizando os falsos positivos, e maximizar os recursos profissionais disponíveis. O Nutriscore avalia a perda de peso recente e diminuição de apetite e, como novidade relativamente às existentes, considera a localização anatómica do cancro e o tratamento a ser realizado (1) Os estudos publicados nos quais foi utilizado o Nutriscore são escassos (2-6). Estes focaram-se na avaliação da sua validade através da sua comparação com o PG SGA, em simultâneo com outras ferramentas como o NRS 2002 e o MUST. O Nutriscore revelou um desempenho igual ou superior, avaliado pela sensibilidade e especificidade (2-5). Para que o Nutriscore possa ser aplicado em Portugal, é necessário que exista uma tradução validada para a língua Portuguesa do original em Inglês. Contudo, esta tradução não foi ainda desenvolvida. O objetivo deste trabalho foi produzir uma tradução do Nutriscore (1) da língua original, o Inglês, para a língua Portuguesa e validar esta tradução. Pretendeu-se também estudar a validade linguística e transcultural - o conteúdo e a equivalência semântica, avaliar o grau de dificuldade de preenchimento, a compreensão, o tempo médio de aplicação e também a aplicabilidade desta ferramenta. 


\section{Aplicação da metodologia e desenvolvimento da versão Portuguesa do Nutriscore}

Este trabalho decorreu entre fevereiro e junho de 2019 nos serviços de Unidade de Dia e Consulta Externa de Oncologia e Nutrição Oncológica da Unidade Local de Saúde do Alto Minho (ULSAM), Entidade Pública Empresarial (EPE).

O Nutriscore é constituído por cinco perguntas de campo fechado (1). Três sobre a perda de peso recente não intencional e alterações de apetite que são respondidas pelo doente e as restantes pelo médico(a), enfermeiro(a) ou nutricionista que aplica o questionário, sobre o local anatómico da patologia e o tratamento a realizar (1).

Para a tradução e validação da versão traduzida foram seguidas as recomendações da International Society for Pharmacoeconomics and Outcomes Research (ISPOR): "Principles of Good Practice for the Translation and Cultural Adaptation Process for Patient-Reported Outcomes (PRO) Measures: Report of the ISPOR Task Force for Translation and Cultural Adaptation", que preconizam os seguintes passos: a preparação, tradução, reconciliação, retrotradução, revisão da retrotradução, harmonização, avaliação da compreensão, revisão, finalização e a produção do relatório final (7).

Este protocolo obteve parecer positivo da Comissão de Ética da ULSAM, EPE (Parecer n. ${ }^{\circ}$ 27/2019), e autorização dos diretores dos serviços envolvidos.

Descrevem-se em seguida os detalhes relativos à aplicação desta metodologia.

1. Preparação: a autora principal da ferramenta, a Sr. ${ }^{\mathrm{a}} \mathrm{Dr}{ }^{\mathrm{a}}$ Lorena Arribas, foi contactada e concedeu permissão para se proceder a este processo. 2. Tradução: dois indivíduos, ambos fluentes em inglês e nativos da língua Portuguesa, desenvolveram duas traduções individuais do questionário original em Inglês para o Português. De modo a atingir o objetivo, as traduções formuladas não eram literais, mas sim conceituais. Isto é, as palavras e termos utilizados eram sinónimos adequados à população geral, tendo em consideração indivíduos com menor literacia, e não simples traduções.

3. Reconciliação: as traduções geradas foram apresentadas a duas nutricionistas com experiência na área da oncologia, as quais identificaram pontos fortes e fracos de cada uma das traduções. Um painel constituído pelo investigador, um nutricionista, um médico e um psicólogo analisou o relatório de ambas, tendo em conta a equivalência do conteúdo (equivalência semântica e sintática) entre a versão original e as traduções e desenvolveu uma versão em que foram integrados os pontos fortes e as sugestões.

4. Retrotradução: procedeu-se à tradução da versão Portuguesa novamente para o idioma original. Neste passo foram criadas duas traduções: uma desenvolvida por um indivíduo nativo da Língua Inglesa e fluente em Português e outra por um Português imigrante em Inglaterra há vinte anos, ambos sem qualquer conhecimento sobre a ferramenta. 5. Revisão da retrotradução: o painel comparou a tradução gerada com a ferramenta original e verificou que existiam três diferenças. Não foi necessário explorar possíveis alternativas pois observou-se a ausência de discrepâncias na equivalência.

6. Harmonização: após revisão, o painel analisou os documentos para encontrar discrepâncias entre todas as traduções produzidas e para as resolver. Com este passo pretendia-se assegurar a equivalência de conceitos entre a versão original e a versão Portuguesa. Após alcançado o consenso, obteve-se a versão pré-final.

7. Avaliação da compreensão ("Cognitive debriefing"): nesta fase iniciou-se o estudo piloto que consistiu na aplicação desta versão pré-final a pessoas doentes, para avaliar a sua compreensão, as dificuldades, o tempo médio de aplicação e a aplicabilidade desta versão traduzida, nos serviços Unidade de Dia, Consulta Externa de Oncologia e de Nutrição Oncológica. Avaliaram-se ainda a aceitabilidade, a relevância e a validade transcultural. Convidaram-se a participar no estudo piloto dezasseis profissionais de saúde na área da oncologia, sendo que apenas seis aceitaram: duas médicas aplicaram um total de dezassete questionários, três nutricionistas aplicaram vinte questionários e nove foram ministrados por um enfermeiro.

A amostra de conveniência foi constituída por 46 doentes (28 do sexo masculino e 18 do sexo feminino), com patologia oncológica, nomeadamente, da bexiga, cólon, estômago, mama, ovário, pâncreas, próstata, pulmão e reto a realizar quimioterapia (QT) e/ou radioterapia (RT) ou tratamento sintomático, em regime de ambulatório. Foram excluídos os indivíduos considerados pelos médicos como não reunindo critérios para realizar tratamento antineoplásico ou como não tendo capacidade cognitiva para compreender e assinar o consentimento informado. Destes 46 doentes, 9 estavam a iniciar/ no início dos tratamentos, 14 encontravam-se a terminar ou tinham acabado há menos de um mês e 21 estavam a meio dos tratamentos. Incluíram-se todos os potenciais participantes que tinham como língua materna a língua Portuguesa e idades iguais ou superiores a dezoito anos. Para a caracterização da amostra, recolheu-se informação relativa à idade, sexo, peso e estatura. Foi cronometrado o tempo de aplicação de cada questionário e não se verificou nenhuma recusa de participação. A percentagem de indivíduos em risco nutricional encontrada foi de $23,9 \%$ e as caraterísticas da amostra encontram-se descritas na Tabela 1. Dos 11 indivíduos identificados em risco nutricional, apenas um não foi referenciado para a consulta de nutrição. Este estava em cuidados paliativos.

\section{Tabela 1}

Caraterísticas dos indivíduos que participaram no estudo piloto

\begin{tabular}{lcc} 
& TOTAL $(\mathrm{n})$ & COM RIsco $n(\%)$ \\
Bexiga & 1 & $0(0 \%)$ \\
\hline Cólon & 15 & $2(4,3 \%)$ \\
\hline Estômago & 8 & $5(10,9 \%)$ \\
\hline Mama & 4 & $1(2,2 \%)$ \\
\hline Ovário & 4 & $0(0 \%)$ \\
\hline Pâncreas & 2 & $2(4,3 \%)$ \\
\hline Próstata & 1 & $0(0 \%)$ \\
\hline Pulmão & 6 & $1(2,2 \%)$ \\
\hline Reto & 5 & $0(0 \%)$ \\
\hline Total & 46 & $11(23,9 \%)$ \\
\hline
\end{tabular}

\begin{tabular}{lcc}
\multicolumn{2}{l}{ COM RISCO } & SEM Risco \\
\hline Idade (anos) & & \\
\hline - Média & 67 & 64 \\
\hline - Máximo & 79 & 82 \\
\hline - Mínimo & 48 & 40 \\
\hline & & \\
\hline IMC $\left(\mathrm{kg} / \mathrm{m}^{2}\right)$ & & \\
\hline - Média & 24,39 & 24,54 \\
\hline - Máximo & 37,81 & 35,90 \\
\hline - Mínimo & 19,91 & 15,19 \\
\hline & & \\
\hline
\end{tabular}

IMC: Índice de massa corporal 
Esta análise permitiu identificar palavras ou expressões que geravam confusão aos profissionais de saúde ou aos utentes. Após terminada a ministração desta versão do Nutriscore, cada um dos seis profissionais de saúde participantes reportou ao investigador principal os pontos fortes e pontos fracos do questionário traduzido, identificando elementos que deveriam ser melhorados e sugeriram possíveis alterações. Estes consideraram a ferramenta de extrema relevância, com validade aparente e boa aceitabilidade por parte dos utentes. Ambas as partes envolvidas a classificaram de alta aplicabilidade, de fácil e rápida aplicação e, ainda, de fácil compreensão. Apenas se verificou dificuldade em entender o significado de "forma involuntária" por parte de três pessoas doentes. O tempo médio de preenchimento do questionário foi de 20 segundos $(n=40)$, com mínimo de 16 segundos e máximo de 1 minuto e 20 segundos.

8. Revisão: o investigador principal reviu os resultados do teste piloto, comparando e analisando a avaliação de cada profissional de saúde para identificar possíveis modificações necessárias. Posteriormente, estas foram discutidas com o painel, não tendo procedido à reformulação de nenhum item, visto que estas iriam para além da tradução e levariam a uma adaptação da ferramenta original.

9. Finalização: a tradução final foi examinada por um professor de
Português para estudar a presença de erros de ortografia, gramática e sintaxe, tendo apenas identificado a ausência de um sinal de pontuação na última frase. O painel considerou então finalizado o processo de tradução e a versão do Nutriscore em Português encontra-se na Figura 1.

10. Produção do relatório final: o englobado no presente documento.

\section{ANÁLISE CRÍTICA}

O presente trabalho permitiu desenvolver com sucesso uma versão em Português da ferramenta de rastreio Nutriscore (1). Esta revelou-se de fácil compreensão, rápida aplicação e apresentou validade aparente. Para estudar a aplicabilidade da ferramenta, a amostra estudada deve ser semelhante à população na qual será aplicada. Deste modo, teria sido mais adequado administrar a ferramenta apenas na Unidade de Dia, visto que é onde se realizam os tratamentos oncológicos. Contudo, por motivos inerentes ao funcionamento do próprio serviço, apenas o enfermeiro-chefe colaborou na investigação, pelo que se alargou o estudo a outras unidades. Apesar desta amostra ser proveniente de um único hospital, trata-se de um hospital distrital, o que terá permitido incluir indivíduos de diversas zonas do distrito e estratos socioeconómicos. Embora não se tenha avaliado o nível

\section{Figura 1}

Versão em Português da ferramenta Nutriscore

NUTRISCORE
\begin{tabular}{ll} 
A. Nos últimos 3 meses, perdeu peso de forma involuntária? \\
\hline Não & $\square(0)$ \\
\hline Não tem a certeza/ não sabe & $\square(2)$ \\
\hline Se sim, quanto peso (em kg) perdeu? & $\square(1)$ \\
\hline $1-5$ & $\square(2)$ \\
\hline $6-10$ & $\square(3)$ \\
\hline $11-15$ & $\square(4)$ \\
\hline$>15$ & $\square(2)$ \\
\hline Não sabe
\end{tabular}

B. Na última semana, tem-se alimentado mal por uma diminuição do apetite?

\begin{tabular}{cc} 
B. Na última semana, tem-se alimentado mal por uma diminuição do apetite? \\
\hline Não & $\square(0)$ \\
\hline Sim & $\square(1)$ \\
\hline
\end{tabular}

\section{LOCALIZAÇÃO / NEOPLASIA}

RISCO NUTRICIONAL

PONTUAÇÃO

Cabeça e pescoço

Trato GI superior: esófago, estômago, pâncreas, intestino

Linfoma que compromete o trato Gl

Pulmão

Abdómen e pélvis: fígado, trato biliar, rins, ovários, endométrio

Mama

Sistema Nervoso Central

Bexiga, próstata

Colorretal

Elevado*

$+2$

Leucemia, outros linfomas

Outros

TRATAMENTO

O utente está a realizar simultaneamente quimioterapia e radioterapia

O utente está a realizar radioterapia hiperfracionada

Transplante de células hematopoiéticas

\begin{tabular}{lcc}
\hline O utente está apenas a realizar quimioterapia & SIM $(+1)$ & NÃO $(+0)$ \\
\hline O utente está apenas a realizar radioterapia & SIM (+0) \\
\hline
\end{tabular}

Outros tratamentos ou tratamentos sintomáticos

${ }^{\star}$ Repetir o rastreio semanalmente em utentes com risco elevado.

Pontuação final:

Pontuação $\geq 5$ : O utente está em risco nutricional. Por favor, encaminhar para um nutricionista. 
de literacia dos mesmos, o Nutriscore foi facilmente compreendido pelos utentes.

A percentagem de indivíduos em risco de desnutrição encontrada no presente estudo, revelou-se menor do que a já descrita na literatura (8), que se poderá justificar pelo recurso a uma amostra de conveniência, pelo não tratamento de doentes patologia de pescoço/cabeça nestas unidades e pelo acompanhamento pelo serviço de Nutrição da maioria dos doentes oncológicos desta instituição.

O processo de tradução, seguindo os princípios da ISPOR (7), permitiu desenvolver a versão em Português do Nutriscore, mantendo os conceitos e equivalência da ferramenta original, quando aplicada a indivíduos portugueses. Esta revelou-se de fácil compreensão e aplicação, com boa aceitabilidade e validade aparente. Conclui-se, assim, que esta ferramenta pode ser útil para determinar o risco de desnutrição em doentes oncológicos em regime de ambulatório em Portugal e eventualmente em outros locais onde seja usada a língua Portuguesa. Espera-se que a disponibilidade da ferramenta auxilie na identificação dos indivíduos em risco de desnutrição e permita iniciar precocemente acompanhamento nutricional, potenciando a terapia medicamentosa e diminuindo os efeitos indesejados.

\section{REFERÊNCIAS BIBLIOGRÁFICAS}

1. Arribas L, Hurtos L, Sendros MJ, Peiro I, Salleras N, Fort E, et al. NUTRISCORE: A new nutritional screening tool for oncological outpatients. Nutrition. 2017;33:297-303.

2. Higuera I, Gonzalez A, Aguado A, Martín M, Sancho S. Evaluation of two nutritional screening methods in oncological patients with active treatment. Clinical Nutrition. 2018;37:S289.

3. Manuella IP, Keila FD, Izabel SDAM, Oliveira STD, Silva FAKD, Rayanne MDAH, et al. Comparison between different nutritional screnning tools in cancer outpatients. Nutricion Clinica y Dietetica Hospitalaria. 2017;37(3):35-43.

4. Aguiar GB. Associação entre a avaliação subjetiva global produzida pelo paciente e a nutriscore em pacientes oncológicos ambulatoriais. 2018. Disponível em: https:// repositorio.ufpe.br/handle/123456789/27645.

5. Álvaro Sanz E, Siles M, Rey Fernández L, Villatoro Roldán R, Rueda Domínguez A, Abilés J. Nutritional risk and malnutrition rates at diagnosis of cancer in patients treated in outpatient settings. Nutrition. 2019;57:148-53.

6. Torres MDG. Importancia del cribado nutricional en el paciente oncológico. Nutricíon Hospitalaria. 2016;33(Supl 6):3-8.

7. Wild D, Grove A, Martin M, Eremenco S, McElroy S, Verjee-Lorenz A, et al. Principles of Good Practice for the Translation and Cultural Adaptation Process for PatientReported Outcomes (PRO) Measures: report of the ISPOR Task Force for Translation and Cultural Adaptation. Value in Health: The Journal of the International Society for Pharmacoeconomics and Outcomes Research. 2005;8(2):94-104.

8. Arends J, Baracos V, Bertz H, Bozzetti F, Calder PC, Deutz NEP, et al. ESPEN expert group recommendations for action against cancer-related malnutrition. Clinical Nutrition. 2017;36(5):1187-96. 